\title{
Editorial
}

\section{Large arteries are more than passive conduits}

The intimate physiological relation between the heart and the arteries has been recognised for hundreds of years. The eighteenth century discovery that resistance to blood flow from the heart resides predominantly in small arteries and arterioles considerably advanced understanding of the integrated circulation. ${ }^{1}$ It became apparent that the cardiac work necessary to maintain a given blood flow depends upon arteriolar tone. The therapeutic importance of this interaction is currently realised in the use of vasodilators to offload the heart in diseases such as heart failure, hypertension, and angina. In contrast, large arteries have generally been considered to have a predominantly passive role in the circulation, because they impose virtually no resistance to blood flow. The concept that large arteries are merely conduits neglects the contribution of their elastic components (elastin, collagen, and smooth muscle) to their stiffness. Because of their elasticity, the large arteries convert pulsatile aortic flow to virtually continuous tissue perfusion by absorbing the energy of pulsatile flow in the elastic components of their walls and releasing this energy during late systole and diastole. If the large arteries were not elastic, the heart would have to accelerate the entire column of blood between the left ventricle and the resistance vessels during early systole, and blood flow would rapidly decelerate to a standstill during diastole. Consequently, the heart would expend more energy in a shorter time and the arteries would be subject to much larger, potentially damaging, physical forces. Large artery elasticity thus makes an important contribution to the overall mechanical efficiency of the circulation which may be particularly relevant in the context of heart muscle disease ${ }^{2}$ or coronary stenosis. ${ }^{3}$

That large arteries impose little resistance to blood flow is not disputed, but the concept of resistance relates primarily to conditions of constant, not pulsatile, flow. Large arteries may oppose pulsations in blood flow while not resisting constant flow; the former effect is determined by arterial elasticity and the latter by arterial diameter. These are fundamentally different properties. The lack of resistance to constant flow posed by the large arteries does not mean that cardiac performance is independent of changes in their mechanical properties.

It seems reasonable to propose that the elastic behaviour of large arteries and thus their contribution to cardiac afterload and the efficiency of perfusion is subject to beat-by-beat control in a manner analogous to the control of arteriolar resistance. The smooth muscle component of the arterial wall makes this possible. Smooth muscle, unlike connective tissue, may change its tone (and therefore its elastic properties) greatly; the magnitude of this phenomenon can be seen during coronary angiography when coronary artery spasm develops. As arterial diameter decreases in more peripheral arteries, the proportion of smooth muscle in the wall and the collagen/elastin ratio increase progressively. These changes result in a progressive increase in stiffness of peripheral vessels and in a potentially greater influence of smooth muscle tone on arterial elasticity. A decrease in smooth muscle tone may be expected to increase the distensibility of an artery and vice versa. This raises the possibility that arterial mechanics may be altered acutely by changes in smooth muscle tone as well as chronically by changes in wall structure. Such changes in arterial mechanical behaviour may be largely independent of changes in resting diameter.

Smooth muscle tone in large arteries is influenced by factors similar to those that control tone in small arteries, such as prostanoids, myogenic responses, and the neurohumoral effects of locally and systemically released sympathetic amines. Increasing physiological and pathophysiological importance is attributed, moreover, to the endothelium. The classic studies by Furchgott and Zawadski, that showed the obligatory role of endothelium in the smooth muscle relaxation by acetylcholine were performed in strips of aorta. ${ }^{4}$ Endothelium-dependent relaxation of smooth muscle in most arteries has subsequently been shown to be mediated by the release of endothelium-derived relaxing factor (EDRF), now known to be nitric oxide. In the microcirculation the ability of EDRF to control and integrate blood flow by transduction of the signals imposed by blood flow (shear stress) and circulating agonists (for example, acetylcholine, ADP, bradykinin) is well recognised. ${ }^{5}$ The physiological consequences of EDRF in controlling the mechanical behaviour of large arteries have, however, been largely ignored. EDRF activity may well be directed towards adjusting the passive elastic properties of the artery wall. Hence, large artery endothelium may directly control the local mechanical properties of the artery wall and thus contribute to the dynamic control of cardiac performance. ${ }^{6}$

It follows that the function of large arteries may be impaired in conditions associated with reduced activity of EDRF. Endothelial dysfunction may thus contribute to arterial stiffening, for example in hypertension, ${ }^{7}$ hypercholesterolaemia, ${ }^{8}$ and heart failure. ${ }^{9}$ This will increase left ventricular load, thereby stimulating hypertrophy, as well as promote atherogenesis. Many treatments, including ACE inhibitors, decrease arterial stiffness as well as dilate small arteries. ${ }^{10}$ Whether restoration of normal endothelial function contributes to these effects remains to be determined. Interventions that reverse endothelial dysfunction (for example L-arginine, fish oil, or exercise) may increase large artery distensibility and thereby promote the overall mechanical efficiency of the diseased circulation.

Two principal problems arise in the design of clinical studies investigating vasomotor control in large arteries. Blood pressure and flow in large arteries are pulsatile and the pressure across the arterial wall itself directly determines its elasticity. ${ }^{11}$ For a simple thin-walled elastic tube, the tension within the wall increases with increasing diameter (by the law of Laplace), but this relation is more complex in a large artery. The wall has finite thickness, its individual constituents have different elastic properties, and resting arterial diameters vary with 
distance from the heart. In an artery composed predominantly of elastin and collagen, load bearing is shifted from elastin to collagen as the artery expands. Since collagen is stiffer than elastin by several orders of magnitude, elastic properties change in a highly non-linear fashion with changes in arterial diameter. The contribution of smooth muscle to local arterial elasticity will depend directly upon its tone, but will depend also upon whether a consequent change in wall tension is borne by the elastin or collagen component. The difficulty in controlling for these confounding variables is perhaps one reason why this important area of circulatory physiology has often been considered the domain of physicists, mathematicians, and fluid dynamicists.

Throughout this discussion we have considered the heart, the large arteries, and small arteries as separate entities with fundamentally different properties. However, they jointly comprise an integrated system. Large arteries affect cardiac performance and cardiac performance will ultimately determine blood flow in small arteries. Microvascular dilatation (induced, for example, by increased local metabolic demand) will reduce peripheral resistance and so increase blood flow throughout the vascular tree, resulting in endotheliummediated dilatation also of large arteries, which thereby become more distensible, unloading the heart and promoting arterial flow. Endothelial dysfunction in large arteries may thus impair the mechanical integration between the heart and the resistance vessels.

In summary, the distensibility of the large arteries is an important determinant of cardiac workload in the integrated circulation. It is likely to be controlled on a beatby-beat basis by changes in the tone of the smooth muscle component of the arterial wall, as induced by locally acting substances including EDRF and by systemic neurohumoral influences. Distensibility is reduced in many disease states that are associated with endothelial dysfunction..$^{78}$ Correction of endothelial dysfunction may provide a novel means of improving cardiac performance by increasing the distensibility of large arteries.

Department of Cardiology,

$M$ W RAMSEY

University of Wales College of Medicine,

Heath Park,

Cardiff CF4 4XN

We thank Professor AH Henderson and Dr RJC Hall for their comments on the paper. MWR and CJHJ are supported by the British Heart Foundation.

1 Hales S. (1733) Statical essays. History of Medicine Series, Library of New York Academy of Medicine, No.22. New York: Hafner Publishing, 1964.

2 Maruyama Y, Nishioka O, Nozaki E, Kinoshita H, Koiwa Y, Takishima $T$. Effects of arterial distensibility on left ventricular ejection in the depressed contractile state. Cardiovasc Res 1993;27:182-7.

3 Watanabe $\mathbf{H}$, Ohtsuka S, Kakihana M, Sugishita Y. Coronary circulation in dogs with an experimental decrease in aortic compliance. $\mathcal{F} \mathrm{Am}$ Coll Cardiol 1993;21:1497-1506.

4 Furchgott FR, Zawadski JV. The obligatory role of endothelial cells in the relaxation of arterial smooth muscle by acetylcholine. Nature 1980;288: relaxation.

5 Henderson AH. Endothelium in control (St Cyres Lecture). Br Heart $f$ 1991;65:116-25.

6 Ramsey MW, Jones CJH, Stewart W, Lewis MJ, Henderson AH. Distensibility of large arteries is increased by endothelium-dependent relaxing factor (EDRF) in normal subjects [abstr]. Br Heart $\mathcal{F}$ 1993;69:

7 Panza J, Quyyumi AA, Brush J, Epstein S. Abnormal endotheliumdependent vascular relaxation in patients with essential hypertension. $N$ Engl ₹ Med 1990;323:22-7.

8 Creager MA, Cooke JP, Mendelson ME, Gallagher SJ, Coleman SM, Loscalzo J and Dzau VJ. Impaired vasodilatation of forearm resistance vessels in hypercholesterolaemic humans. $\mathcal{F}$ Clin Invest 1990;86:228-34.

9 Kaiser L, Spickard RC, Olivier NB. Heart failure depresses endotheliumdependent responses in canine femoral artery. Am $\mathcal{Y}$ Physiol 1989;256: H962-H969.

10 Safar ME, Levy BI. The response of large arteries to antihypertensive treatment: II Pharmacological aspects. In: O'Rourke MF, Safar ME, Dzau VJ, eds. Arterial vasodilatation. Mechanisms and therapy. London: Edward Arnold, 1993.

11 Milnor WR. Hemodynamics. Baltimore: Williams and Wilkins, 1989. 